\title{
Oral Cancer: Incidence and Management
}

\author{
Ivan Minić* and Ana Pejčić \\ Department of Periodontology and Oral Medicine, Medical Faculty, University of Nis, Serbia
}

\begin{abstract}
Oral cancers are among the 10 most common tumors in Europe and the United States. The major recognized risk factors include smoking and alcohol what is possible explanation for higher incidence among men. The risk of developing a tumor of the oral cavity is 38 times higher in the population that consuming alcohol and cigarettes. Cancer of the oral cavity is a serious disease and only half of the patients survive the next five years. According to the World Health Organization classification, oral cancer includes malignant tumors in the lips area, anterior two thirds tongue, gingiva, bottom of the oral cavity, retromolar triangle, hard palate and mucous membranes of the oral cavity. In spite of the advancement of medicine, oral cancer remains a malignant tumor with a high mortality rate. The reason for this was primarily enhanced by the tumor stage at the time of diagnosis. On the other hand, the oral cancer is a tumor that can be detected by a simple examination of the oral mucosa at an early stage, before it starts to cause symptoms.
\end{abstract}

Keywords

Oral cancer, Incidence, Management, Dentistry

\section{Introduction}

Oral cancers make up 2 to $5 \%$ of all cancers [1]. Oral cancers are among the 10 most common tumors in Europe and the United States [2]. The average incidence in Europe is 10 toward hundred thousand inhabitants. Oral cancers account for $30 \%$ of all head and neck carcinoma [3].

According to WHO Cancer Register there were an estimated three hundred and fifty thousand new cases diagnosed and fifty thousand deaths recorded in 2017 [4]. In all countries of the world, oral cancers is more common in men (8 to 10 toward 1) [5]. The highest frequency is recorded in the Caribbean region, in eastern and southeastern Asia, and in some European countries [6-8].

The major recognized risk factors include smoking and alcohol what is possible explanation for higher incidence among men [9]. The risk of developing a tumor of the oral cavity is 38 times higher in the population that consuming alcohol and cigarettes [10]. Although the diagnostic and therapeutic possibilities of patients with oral cancer significantly advanced, the mortality rate remained high (around 50\%) and has not improved over the last 30 years [11].

Cancer of the oral cavity is a serious disease and only half of the patients survive the next five years [12]. Cancer of the oral cavity is in most cases detected in the advanced stages, and the five-year survival is less than $50 \%$ [11]. Treatment success and length of survival increase significantly if the diagnosis is made in the early stage of the disease [13].

According to the World Health Organization classification, oral cancer includes malignant tumors in the lips area, anterior two thirds tongue, gingiva, bottom of the oral cavity, retromolar triangle, hard palate and mucous membranes of the oral cavity. This does not include neoplasms of the glands, tonsils, and large salivary glands [14].

The soft and hard palate compound represents the upper, and the line of large papillae on the base of the tongue is the lower limit of the localization of oral and pharyngeal carcinoma [15].

Early signs of oral cavity cancer are red or whitish lesions of the mucous membrane that persist, a nonhealing ulcer, an island or thickening of the structure of the oral cavity, atypical changes in the mucous

*Corresponding author: Ivan Minić, Department of Periodontology and Oral Medicine, Medical Faculty, University of Nis, Blvd. Nikole Tesle 63/8, 18000 Nis, Serbia, Tel: +381643004883

Accepted: December 04, 2018

Published online: December 06, 2018

Citation: Minić I, Pejčić A (2018) Oral Cancer: Incidence and Management. J Oral Cancer Res 2(1):23-27 
membrane, a toothache without a clear cause, unexplained bleeding from the gingiva or nose.

Late signs are induction of the affected area, paraesthesia, of tongue and lips, stiffness of the jaw, dysphagia, dyspnoea, visual impairment, pain of the ears and enlarged lymph nodes on the neck [16-22].

Although the oral cavity is readily available for review, research shows that a quarter of patients are late with the onset of treatment. The late diagnosis of oral cancer is influenced by at least 3 factors: Low incidence, lack of knowledge and awareness of oral cavity cancer in the population and among healthcare workers, and difficulties in the health system that make patients and dentists difficult access to patients [23]. Additional negative predictors are older age, male gender, poorer education and poorer socioeconomic status.

People with low risk of oral cancer are non-smokers and non-alcoholic drinkers, people under the age of 40 , and those who have no oral cancer or precancerous lesions in their personal and family history and who have developed awareness of oral cancer and regularly go to dental reviews [24,25].

\section{Risk Factors for Oral Cancer}

\section{Cigarette smoking}

Numerous studies have demonstrated a clear connection between oral cancer and tobacco use by smoking or chewing.

The use of smokeless tobacco increases the risk of oral cavity cancer by 3-4 times, while smoking tobacco is even more dangerous and increases the risk by 3-17 times in relation to non-smoking, depending on the amount of cigarettes smoked. The proportion of smokers among people with oral cavity cancer is greater than $80 \%$. This clearly shows that smokers are high-risk populations to be included in prevention and screening programs for early detection of oral cavity cancer [26].

\section{Alcohol}

Epidemiological studies on smokers have shown that alcohol additionally increases the risk of cancer several times, and this fact should be emphasized in antismoking and alcohol campaigns as well as in screening programs for early detection of oral cancer [27].

\section{Human papilloma virus (HPV)}

There is increasing evidence that confirms the role of HPV in the development of oral cavity cancer. Two recent meta-analyzes have found that the HPV-16 is an independent prognostic factor for oral cancer. It is believed that the proteins E6 and E7 that secretion the infected cells change the function of suppression oncogenes $\mathrm{p} 53$ and $\mathrm{pRb}$ resulting in uncontrolled DNA replication and impairment of apoptosis. The combined effect of these mechanisms leads to malignant transformation of normal epithelial cells [28-30].

Human papilloma virus is transmitted sexually. Therefore, it is necessary to emphasize the need for changes in sexual behavior, especially the use of condoms and the avoidance of oral sex.

\section{Poor diet}

Nutrition poor with fresh fruits and vegetables present a significant risk for the onset of oral cancer. Studies have shown that the addition of beta-carotene or vitamin A to the diet reduces the risk of premature and malignant changes in the oral cavity [31].

\section{Ultraviolet (UV) radiation}

UV radiation is the main cause of oral cancer, which accounts for $30 \%$ of the total number of cases of oral cavity cancer. High incidence of oral cancer is present in white, especially male smokers exposed to the sun during outdoor work. Cancer occurs more often on the lower lip, and can be preceded by actin heliitis for years [32].

\section{Hygiene of the oral cavity and chronic inflamma- tion}

It has been proven that some bacteria and fungi in the oral cavity can convert alcohol into acetaldehyde that is known to be carcinogenic and thus trigger the onset of malignant changes. Some evidence of the negative role of mouthwash in alcohol-based preparations is also supported [33].

\section{Clinical Picture}

Oral cancer at an early stage does not cause any problems and symptoms begin only in advanced stages of the disease. In the initial stages of oral cancer, the cavity is manifested as erythroplakia, a red-colored lesion or red-and-white, sharply limited by surrounding tissue. Over time, there is the development of ulcers with elevated edges. Oral cancer can also have an exophytic form, and then it is manifested by a mass that fills the oral cavity.

It usually occurs at the edges of the tongue, the floor of the mouth cavity, the retromolar region, but also on the other parts of the oral cavity can be appear [34-37].

The most common discomfort that patients come to the doctor is discomfort and burning in the mouth. It can also cause difficulty and painful swallowing, difficulty speaking, swinging of the teeth, bleeding from the oral 
cavity, and ear pain. Rarely, the first sign of cavity of the oral cavity may be the appearance of one or more nodes on the neck. In over $50 \%$ of patients diagnosed, the disease is in an advanced stage.

Carcinoma of the oral cavity is most often metastasized to the cervical lymph nodes, while distant metastases are rare. The most affected are this nodes: submandibular, digastric and upper cervical nodes located on the same side of the neck as the tumor.

\section{Early Detection of Oral Cancer}

Carcinoma of the oral cavity can be detected at the earliest stage by simply examining the oral mucosa. It is important to point out that these are localizations that are accessible to the naked eye examination and visible during a regular dental examination. The review is noninvasive, takes 1-1.5 minutes, does not require any special equipment and is not unpleasant for the patient.

In addition to a better knowledge of the physiological and pathological characteristics of the oral mucous membrane, the dentist is in an ideal position to perform daily examinations for early detection of oral cavity cancer since he regularly looks at the patient's mouth in the nature of his work.

Since oral cancer does not cause symptoms at an early stage, dentists should warn of the need for regular examination of oral mucous membranes for all patients, regardless of whether they have a patient's problems or not. This applies particularly to high-risk groups - men, smokers, over 40 who regularly consume alcohol.

Changes in the mucous membrane of the oral cavity that are susceptible to early-stage oral cavity are [1621]:

- Red lesion (erythroplakia)

- Red-white lesion (erythroleukoplakia)

- Changing the texture of the mucous membrane - the appearance of velvety (granular) surface

- Ulceration that does not heal more than 14 days

In the event of such changes, it is necessary to instruct the patient for further treatment to a specialist who has experience with pathological changes in the oral mucosa (oral pathologist, oral surgeon), since other pathological changes in the oral mucous membranes can also manifest in a similar way [22]. All wounds, red, red and white or white changes in the unclear cause of the mucous membrane mucous membranes that do not complete after 2-3 weeks should be treated for the purpose of excluding the cancer. This also applies to all lumps and tissue enlargement in the oral cavity or neck. There are many more common and benign causes of such occurrences, but it is recommended to review the specialist, especially if the changes last longer than the specified time [23].

It is important to emphasize that these changes should not be painful because oral cancer in the earlier stages usually does not cause painful symptoms, although the pain does not exclude it. Furthermore, difficulties in chewing, swallowing, sudden tooth decay, and harshness are the reasons for talking to a dentist or doctor [24].

Some of the observed changes in the oral cavity will be diagnosed by the dentist as the so-called. potentially malignant oral lesions. These changes are not cancer, but they have a higher likelihood of transition to cancer than in normal mucous membranes. Such changes will be closely monitored and regularly monitored by a dentist, especially a specialist in oral medicine, in order to react in time. In addition to the education of patients and dentists, screening programs are very important. "The American Cancer Society" recommends conducting screening every three years in patients who have no symptoms and who are aged between 20 and 40-years-old, while they are older patients who were also symptom free should be examined every year [25]. Patients who smoke and consume alcohol are considered high-risk patients and they would should be included in the screening program every year, regardless of age. Although the visual examination is still a gold standard in the discovery of oral cancer, In recent years more and more new screening methods have been introduced. Some of these methods are florescent visualization using a device called VEL scope and which notes the changes in normal autofocusence associated with the changes which arise in the tissue of carcinogenesis, then the use of toluidine blue, markers from saliva and genetic profusion of patients [26-28].

The worst that can be done is to leave one of the previously mentioned changes unattended. A specialist will, if indeed it is a suspected formation, take a biopsy to confirm the final diagnosis and promptly refer the patient to treatment [29].

If the existence of pre-cancerous changes in the oral mucous membrane is established, the patient will be placed under intensive supervision involving specialist examinations 2-4 times a year [30].

Regular controls of patients with oral precancerous can be intervened when the earliest signs of malignant transformation occur.

\section{Conclusion}

In spite of the advancement of medicine, oral cancer remains a malignant tumor with a high mortality rate. 
The reason for this was primarily enhanced by the tumor stage at the time of diagnosis. On the other hand, the oral cancer is a tumor that can be detected by a simple examination of the oral mucosa at an early stage, before it starts to cause symptoms.

The inspection is performed with a bare eye, without the use of special equipment and takes about 1 minute. Due to the nature of their work, dentists are in the ideal position to examine their oral mucosa to all their patients and to send them to specialist treatment in case of any suspected changes. A timely diagnosis and treatment of oral cancer can significantly improve the prognosis and quality of life of these patients.

\section{References}

1. Kademani D (2007) Oral cancer. Mayo Clin Proc 82: 878-887.

2. (2007) American Cancer Society.

3. (2004) The world health report 2004: Changing history. World Health Organization.

4. (2017) Oral health: Action plan for promotion and integrated disease prevention. World Health Organization.

5. Vissink A, Burlage FR, Spijkvert FKI, et al. (2003) Prevention and treatment of the consequences of head and neck radiotherapy. Crit Rev Oral Biol Med 14: 213-225.

6. Rogers SN, Vedpathak SV, Lowe D (2011) Reasons for delayed presentation in oral and oropharyngeal cancer: The patients perspective. Br J Oral Maxillofac Surg 49: 349-353.

7. Jamison DT, Breman JG, Measham AR, et al. (2006) Disease control priorities in developing countries. $\left(2^{\text {nd }} e d\right)$, Washington: The World Bank/Oxford University Press.

8. Hecht SS, Szabo E (2014) Fifty years of tobacco carcinogenesis research: From mechanisms to early detection and prevention of lung cancer. Cancer Prev Res (Phila) 7: 1-8.

9. Hertrampf K, Wenz HJ, Koller M, et al. (2011) Comparing dentists' and the public's awareness about oral cancer in a communitybased study in Northern Germany. J Craniomaxillofac Surg 40: 28-32.

10. Applebaum E, Ruhlen TN, Kronenberg FR, et al. (2009) Oral cancer knowledge, attitudes and practices: A survey of dentists and primary care physicians in Massachusetts. J Am Dent Assoc 140: 461-467.

11. Ryan K, Hegarty AM, Hodgson T (2014) Aetiology, diagnosis and treatment of oral lichen planus. Br J Hosp Med (Lond) 75: 492-496.

12. Martín-Hernán F, Sánchez-Hernández JG, Cano J, et al. (2013) Oral cancer, HPV infection and evidence of sexual transmission. Med Oral Patol Oral Cir Bucal 18: 439-444.

13. Ferlay J, Soerjomataram I, Dikshit R, et al. (2015) Cancer incidence and mortality worldwide: Sources, methods and major patterns in GLOBOCAN 2012. Int J Cancer 136: E359-E386.

14. Radoï L, Luce D (2013) A review of risk factors for oral cavity cancer: The importance of a standardized case definition. Community Dent Oral Epidemiol 41: 97-109.

15. (2002) National cancer control programmes: Policies and managerial guidelines: Executive summary. World Health Organization.
16. Benson E, Li R, Eisele D, et al. (2014) The clinical impact of HPV tumor status upon head and neck squamous cell carcinomas. Oral Oncol 50: 565-574.

17. Chi AC, Day TA, Neville BW (2015) Oral cavity and oropharyngeal squamous cell carcinoma - an update. CA Cancer J Clin 65: 401421.

18. McCullough MJ, Prasad G, Farah CS (2010) Oral mucosal malignancy and potentially malignant lesions: An update on the epidemiology, risk factors, diagnosis and management. Aust Dent J 55: 61-65.

19. D'Souza G, Kluz N, Wentz A, et al. (2014) Oral Human Papillomavirus (HPV) infection among unvaccinated high-risk young adults. Cancers 6: 1691-1704.

20. Chinn SB, Myers JN (2015) Oral cavity carcinoma: Current management, controversies, and future directions. J Clin Oncol 33: 3269-3276.

21. Ding $Y$, Yao H, Yao $Y$, et al. (2013) Protection of dietary polyphenols against oral cancer. Nutrients 5: 2173-2191.

22. Warnakulasuriya S, Fennell N, Diz P, et al. (2015) An appraisal of oral cancer and pre-cancer screening programmes in Europe: $A$ systematic review. J Oral Pathol Med 44: 559-570.

23. Adrien J, Bertolus C, Gambotti L, et al. (2014) Why are head and neck squamous cell carcinoma diagnosed so late? Influence of health care disparities and socio-economic factors. Oral Oncol 50: 90-97.

24. Stefanuto P, Doucet JC, Robertson C (2014) Delays in treatment of oral cancer: A review of the current literature. Oral Surg Oral Med Oral Pathol Oral Radiol 117: 424-429.

25. Varela-Centelles $P$, López-Cedrún JL, Fernández-Sanromán J, et al. (2017) Key points and time intervals for early diagnosis in symptomatic oral cancer: A systematic review. Int J Oral Maxillofac Surg 46: 1-10.

26. Iriti M, Varon EM (2013) Chemopreventive potential of flavonoids in oral squamous cell carcinoma in human studies. Nutrients 5 : 2564-2576.

27. Poh CF, Zhang L, Anderson DW, et al. (2006) Fluorescence visualization detection of field alterations in tumor margins of oral cancer patients. Clin Cancer Res 12: 6716-6722.

28. Pereira L, Braga Caputo J, Midori Castelo P, et al. (2015) Oral physiology and quality of life in cancer patients. Nutr Hosp 31: 2161-2166.

29. Kosicki DM, Riva C, Pajarola GF, et al. (2007) Oral CDx brush biopsy - a tool for early diagnosis of oral squamous cell carcinoma. Schweiz Monatsschr Zahnmed 117: 222-227.

30. Devi S, Singh N (2014) Dental care during and after radiotherapy in head and neck cancer. Natl J Maxillofac Surg 5: 117-125.

31. Bernier J, Cooper JS, Pajak TF, et al. (2005) Defining risk levels in locally advanced head and neck cancers: A comparative analysis of concurrent postoperative radiation plus chemotherapy trials of the EORTC (\#22931) and RTOG (\# 9501). Head Neck 27: 843850.

32. Shrime MG, Gullane PJ, Dawson L, et al. (2010) The impact of adjuvant radiotherapy on survival in T1-2N1 squamous cell carcinoma of the oral cavity. Arch Otolaryngol Head Neck Surg 136: $225-228$

33. Luryi AL, Yarbrough WG, Niccolai L, et al. (2014) Public awareness of head and neck cancers: A cross-sectional survey. JAMA Otolaryngol Head Neck Surg 140: 639-646. 
34. Villa A, Kreimer AR, Pasi M, et al. (2011) Oral cancer knowledge: A survey administered to patients in dental departments at large Italian hospitals. J Cancer Educ 26: 505-509.

35. Hertrampf K, Wenz HJ, Koller M, et al. (2013) Early detection of oral cancer: Dentists' opinions and practices before and after educational interventions in Northern-Germany. J Craniomaxillofac Surg 41: e201-e207.
36. Saleh A, Kong YH, Haron N, et al. (2016) Oral cancer screening in private dental practices in a developing country: Opportunities and challenges. Community Dent Oral Epidemiol 45: 112-119.

37. Omana-Cepeda C, Jané-Salas E, Estrugo-Devesa A, et al. (2016) Effectiveness of dentist's intervention in smoking cessation: $A$ review. J Clin Exp Dent 8: e78-e83. 